\title{
Development of a Generalized Corcos model for the prediction of turbulent boundary layer induced noise
}

\author{
Anna Caiazzo ${ }^{1,2}$, Roberto D'Amico *1, and Wim Desmet ${ }^{1,2}$ \\ ${ }^{1}$ KU Leuven, Department of Mechanical Engineering, Division PMA \\ Celestijnenlaan 300B - box 2420, B-3001 Heverlee, Belgium \\ ${ }^{2}$ Member of Flanders Make \\ e-mail: anna.caiazzo@kuleuven.be,wim.desmet@kuleuven.be
}

The wall pressure fluctuations induced by turbulent boundary layers (TBL) constitute a fundamental source of aerodynamic noise. Their study is receiving a significant amount of attention, especially when considering the need for improved vehicle comfort. The action of the stochastic pressure distribution associated with turbulence excites the vehicle structure, generating vibration and subsequent acoustic radiation into the acoustic passenger cavity. In such a situation, a good description of the wall pressure field is necessary for an accurate prediction of the vibration and noise propagation [1]. In particular, in early design stage, simple TBL models are an ideal choice to account for turbulent excitation because they lead to quick understanding and efficient computation. In this work the stochastic source reconstruction is considered as an alternative to methods based on CFD simulations. As proposed by semi-empirical models of TBL, fits to experimental data are used to describe the pressure loading. Such TBL models are very useful in the early design stage even though they are accurate only for a few simple configurations of the structure that allow assimilating vehicle parts to flat plates. Among the existing mathematical models of TBL wall pressure fluctuations, the Corcos model is the most popular for its advantageous mathematical features and its accurate description of the wavenumber-frequency spectrum of the wall pressure in the convective domain. However, its major drawback is the small rate of decay for wavenumbers below the coincidence frequency where experimental evidence suggests that it tends to overpredict the spectrum even up to $20 \mathrm{~dB}$. On the contrary, other models such as Chase or Smol'yakov-Tkachenko are more accurate in that range, but they lack simplicity in the mathematical descriptions, with a significant impact on the computation time. This makes the Corcos model very versatile, besides being the reference for other models [1].

In this paper a new model for turbulent boundary layer excitation in vibro-acoustic problems is proposed [2]. As it generalizes the widely used Corcos model, it is called Generalized Corcos. Such a model is based on a two-dimensional Butterworth function rather than on a Lorentzian one as in the classic Corcos [3, 4]. From the latter, it preserves the main advantages, namely, simple mathematics and a closed form solution. But at the same time, by properly choosing the order of the Butterworth filter along the two directions, $n$ along the streamwise direction $x$ and $m$ along the spanwise direction $z$, it is possible to reduce the Corcos spectrum in the entire wavenumber range outside the convective ridge or, more generally, to control the decay of the TBL model. This means an improvement of the accuracy at low wavenumbers over the original Corcos model [2]. The normalized wavenumber-frequency spectra $\tilde{\Phi}_{p p}(\mathbf{k} ; \omega)$ of the models of Corcos, Chase, Smol'yakov-Tkachenko and Generalized Corcos are shown in figure 1-a, where $\mathbf{k} \equiv\left(k_{x}, 0, k_{z}\right)$ is the twodimensional wavevector and $\omega$ is the radian frequency. The spectral levels are plotted with dimensionless longitudinal wavenumber $U_{c} k_{x} / \omega$, for $k_{z}=0$ with the convective velocity, $U_{c}=0.7 U_{\infty}$. In figure 1-b, the

\footnotetext{
${ }^{*}$ Currently working at Autoneum, Switzerland
} 


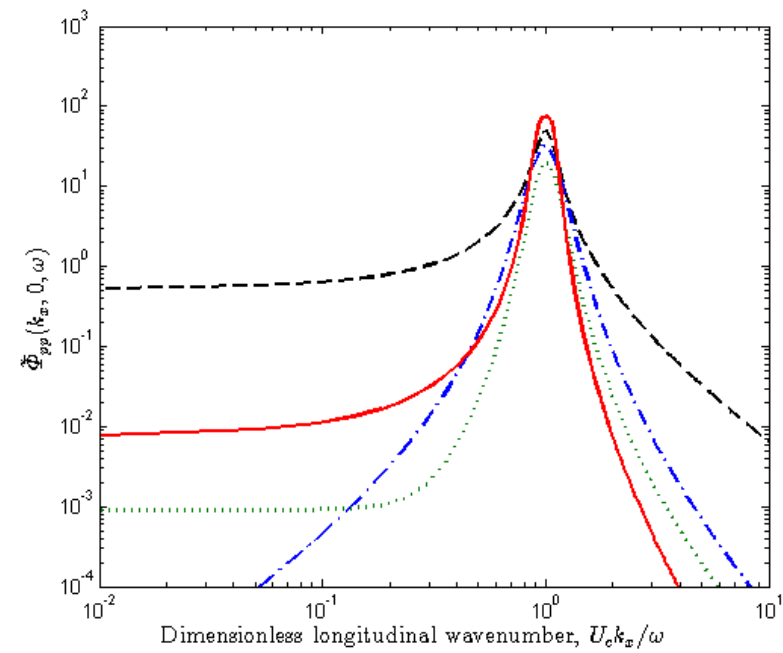

(a)

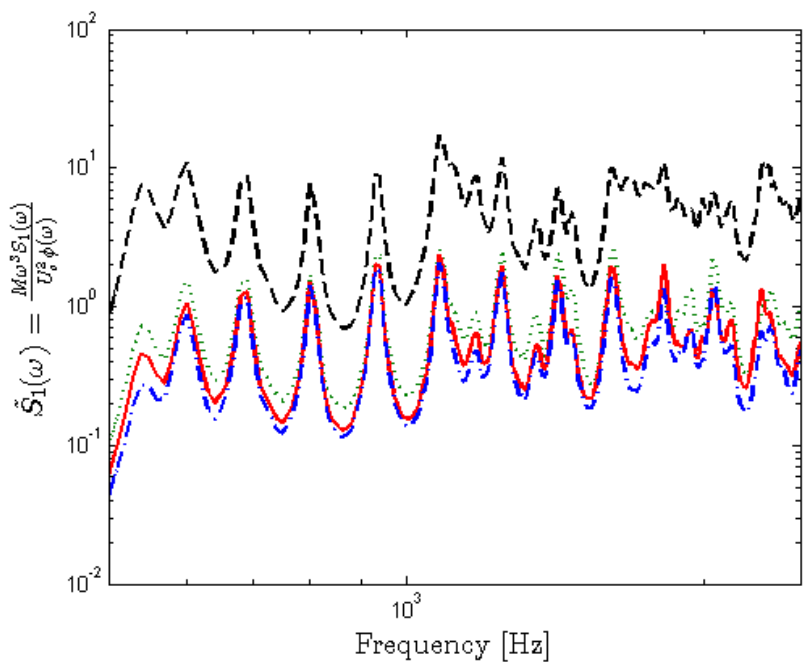

(b)

Figure 1: Normalized Wavevector Spectrum in $k_{x}$ for $S h=24.8$ at $500 \mathrm{~Hz}$; (b) Radiated sound power

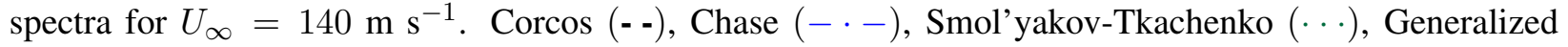
Corcos with $m=1$ and $n=2(-)$.

effect of the choice of a different model of TBL on the sound power radiated, $\tilde{S}_{1}(\omega)$, by a simply supported rectangular panel is shown for $U_{\infty}=140 \mathrm{~m} \mathrm{~s}^{-1}$. In figure $1, m$ is fixed equal to one as found in the Corcos model and $n$ is increased to 2 , in order to see the improvement with respect to the classic Corcos [2, 5]. Indeed, in figure 1-b, the low-wavenumber spectra seen for the Generalized Corcos model lie at a lower radiated sound power than Corcos, between Chase and Smol'yakov-Tkachenko, which means an improved accuracy at low-wavenumbers over the original Corcos model. Besides the analytical development of the proposed model, the paper presents some application cases to benchmark the proposed findings against the most used models. Furthermore, a more detailed analysis on the effect of different orders of the Butterworth filter is addressed. Future research will focus on further investigating the potential of the proposed model and extending its application to more complex cases.

\section{References}

[1] E. Ciappi, S. De Rosa, F. Franco, J.-L. Guyader, and S. A. Hambric, Flinovia - Flow Induced Noise and Vibration Issues and Aspects. Springer, 2015.

[2] A. Caiazzo, R. D’Amico, and W. Desmet, "A Generalized Corcos model for modelling turbulent boundary layer wall pressure fluctuations," Journal of Sound and Vibration, vol. 372, pp. 192-210, 2016.

[3] Roberto D'Amico, Efficient frequency averaging techniques for noise and vibration simulations. $\mathrm{PhD}$ thesis, KU Leuven, 2014.

[4] R. D'Amico, K. Koo, D. Huybrechs, and W. Desmet, "On the use of the residue theorem for the effcient evaluation of band-averaged input power into linear second-order dynamic systems," Journal of Sound and Vibration, vol. 332, no. 26, pp. 7205-7225, 2013.

[5] A. Caiazzo, R. D. Amico, and W. Desmet, "Use of a Generalized Corcos model to predict flow-induced noise in a cavity-plate system," in ISMA2016 \& USD2016, (Leuven, Belgium), 2016. 